\title{
A novel method for the analysis of drug- resistant phenotypes of hepatitis $B$ virus
}

\author{
BO QIN ${ }^{1,2}$, TINGTING HE ${ }^{1}$, ZHIJUN CHEN $^{1}$, WENYING XU ${ }^{1}$, GUOSHAO PAN $^{1}$ and CHUNYU TU ${ }^{1}$ \\ ${ }^{1}$ Shaoxing Centre for Disease Control and Prevention, Shaoxing, Zhejiang; ${ }^{2}$ State Key Laboratory of Virology, \\ Wuhan Institute of Virology, Chinese Academy of Sciences, Wuhan, Hubei, P.R. China
}

Received November 12, 2012; Accepted January 14, 2013

DOI: $10.3892 /$ ijmm.2013.1277

\begin{abstract}
Chronic hepatitis B virus (CHB) infection is a major cause of cirrhosis and hepatocellular carcinoma. Nucleoside analogs (NAs) are popularly used to treat chronic hepatitis B virus (HBV) infections; however, the anti-HBV effect is attenuated by drug-resistant viral mutations selected during long-term antiviral therapy. The timely analysis of drugresistance mutations is essential in order to adjust treatment regimes. In this study, a T1699C substitution was introduced into the $x$ gene of pHBV1.3 to generate an additional XhoI site, termed pHBV1.3-XhoI, which is a nonsense mutation and does not influence protein expression, HBV replication ability, or NA susceptibility. Based on co-transfection with weak or nonreplicative HBV plasmids and $\mathrm{pHBV} 1.3-\mathrm{XhoI}$ or $\mathrm{pHBV} 1.3$ and -XhoI-P-null plasmids into hepatocellular carcinoma cells, PCR was used to amplify 1176-bp segments of T/C1699 using the isolated HBV encapsulated DNA as a template, modified by XhoI digestion and subjected to agarose gel electrophoresis. Different bands composed of different virions were used to distinguish the replication capacities of the plasmids. Our results demonstrated no significant effects when different virions co-existed. A novel resistance test method was developed by co-transfection with pHBV1.3-XhoI and -rtL180M/M204V and treatment with various NA concentrations. Different bands composed of pHBV1.3-XhoI or -rtL180M/M204V were used to distinguish NA susceptibility. The bands composed of pHBV1.3 were more sharply reduced by lamivudine (LMV) than -rtL180M/M204V. The data demonstrate that the method established in our study may be used for the analysis of drug-resistant phenotypes at the cellular level.
\end{abstract}

\section{Introduction}

Hepatitis B virus (HBV) is a significant human pathogen that causes serious health problems (1) and chronic HBV (CHB)

Correspondence to: Dr Bo Qin, Shaoxing Center for Disease Control and Prevention, Shaoxing, Zhejiang 312071, P.R. China E-mail: qinbo0809@hotmail.com

Key words: hepatitis B virus, quasispecies, nucleos(t)ide analogues, drug-resistant phenotype analysis, co-transfection infections affect more than 350 million individuals worldwide (2), leading to the development of cirrhosis and hepatocellular carcinoma (3). HBV is a hepadnavirus and replicates through reverse transcription of an RNA intermediate.

Until now, several nucleos(t)ide analogues (NAs), including lamivudine (LMV), adefovir dipivoxil (ADV), entecavir (ETV), telbivudine (LdT) and tenofovir (TDF), have been approved for the treatment of CHB and are powerful inhibitors of HBV replication $(4,5)$. In vivo and in vitro, NAs mimic physiological nucleosides in terms of uptake and metabolism and are incorporated into newly synthesized DNA chains (6), leading to synthesis inhibition through termination of DNA replication $(7,8)$.

In $\mathrm{CHB}$ patients, $\mathrm{HBV}$ exists in the form of quasispecies due to the error-prone HBV reverse transcriptase (RT), which is responsible for the rich variety of drug-resistant mutants $(9,10)$. HBV RT is subdivided into 7 domains ( $G, F$ and A-E), in which nearly all resistance mutations are located. NA-resistant mutations inhibit the anti-HBV effects of NAs and induce virological advancement and hepatopathological progression (5). A number of mutations have been reported to account for drug resistance. Mutations of rtM204I or rtM204V in the YMDD motif within the RT domain of the HBV polymerase lead to LMV resistance (LMVr) and LdT resistance (LdTr) $(11,12)$ and they are usually accompanied by compensatory mutations of rtL180M and/or rtV173L that restore HBV replication capacity (13). ETV resistance (ETVr) has also been observed in patients with LMVr (14). Under the background of rtM204I/V, a combination of mutations in the B, $\mathrm{C}$ or $\mathrm{D}$ domains of RT may result in ETVr $(15,16)$. The substitution mutations rtA181V/T or $\mathrm{rtN} 236 \mathrm{~T}$ can reduce the anti-HBV effects of ADV, while none have been reported to induce TDF resistance, apart from rtV214A, rtQ215S and rtA194T, although they require further confirmation $(5,17,18)$. Usually, when LMVr occurs, ADV remains effective $(19,20)$. Due to a higher genetic barrier to HBV resistance than LMV and ADV, ETV and TDF are high potency drugs for the treatment of $\mathrm{CHB}$ patients, even those with LMVr and ADVr (21).

Due to the broad spectrum of therapeutic options for antiHBV therapy, HBV replication can be effectively inhibited in the absence of drug-resistance. Therefore, it is crucial to identify mutations through drug-resistant phenotype analysis and adjust treatments accordingly. In this study, an additional restriction site (XhoI) was introduced into $\mathrm{pHBV1.3}$ to produce 
pHBV1.3-XhoI, which was distinguished from mutants without the XhoI site via PCR amplification and XhoI digestion after co-transfection. The T1699C substitution had no effect on the HBV replication level, antigenic protein production and secretion, or drug-sensitivity. Hence, pHBV1.3-XhoI can completely replace $\mathrm{pHBV} 1.3$ for drug-resistance research. Different virions were shown to co-exist in a single cell, which was achieved by co-transfection to closely resemble actual quasispecies. The present study provides simple, practicable and adjustable methods for mimicing quasispecies for the identification of progeny viruses. Based on these methods, we addressed the co-existence of mutant (MT) and wild-type (WT) virions in individual cells and analyzed resistant MT phenotypes using novel methods. In the future, we aim to put this method into practice to discover resistance-related mutations and clarify whether the mutations affect NA susceptibility in vitro and in vivo.

\section{Materials and methods}

Plasmid constructs. pHBV1.3-MTs (Fig. 1A) with C669A/ A741G/T1699C/T2310C substitutions were introduced into the HBV genome by PCR-based mutagenesis using the primer pairs carrying specific mutations (Table I) and pHBV1.3 (pBluescript $\mathrm{KS}^{+}$backbone) as the template, a replication-competent plasmid with 1.3-fold over-length genotype A genome (GenBank accession no. U95551, ayw) $(22,23)$. The plasmids (pHBV1.3rtM204V/I) were constructed in our previous study (24).

NAs. Five types of NAs were used in this study, including LMV (3TC; GlaxoSmithKline, Middlesex, UK), ADV (Gilead Sciences, Middlesex, UK), ETV (Bristol-Myers Squibb Co., New Brunswick, NJ, USA), LdT (Novartis Pharmaceuticals Canada, Inc., Dorval, QC, Canada), and TDF (Gilead Sciences), which are all commercial pills that were ground, dissolved in appropriate solutions as per the manufacturers' instructions, and filtrated with $0.22-\mu \mathrm{m}$ filters (Millipore Carrigtwohill, County Cork, Ireland). Based on the molecular weights and masses of each drug, the concentrations of the stock solutions were estimated and diluted to the required concentrations. The drugs were added to the assay medium at the indicated concentrations.

Cell culture and transfection. Huh7 cells, a hepatocellular carcinoma cell line, were cultured in Dulbecco's modified Eagle's medium (DMEM; Invitrogen, Carlsbad, CA, USA) at $37^{\circ} \mathrm{C}$ in a $5 \% \mathrm{CO}_{2}$ atmosphere supplemented with $10 \%$ fetal bovine serum (FBS; Gibco, Carlsbad, CA, USA), 2 mM/l of glutamine, $100 \mathrm{IU} / \mathrm{ml}$ of penicillin, and $100 \mathrm{IU} / \mathrm{ml}$ of streptomycin (25). Huh7 cells were seeded in 6-well plates and transfected with $2 \mu \mathrm{g}$ of plasmids per well using Lipofectamine 2000 (Invitrogen) with or without the indicated concentration of NAs (4). For transfection assays, HBV-bearing plasmids were co-transfected with a SEAP expression vector into Huh7 cells, the relative efficiency of transfections was assessed by measuring SEAP activity in the culture medium using a chemiluminescent detection method (26).

Enzyme-linked immunosorbent assay (ELISA). Huh7 cells were transfected with the indicated HBV-bearing plasmids, hepatitis B surface antigen (HBsAg), and hepatitis B e antigen (HBeAg) in the supernatant at $72 \mathrm{~h}$ post-transfection and were detected using the appropriate diagnostic kits (Shanghai Kehua Bio-Engineering Co., Ltd., Shanghai, China) according to the manufacturer's instructions.

Quantitative real-time PCR ( $q R T$-PCR). DNase I (Roche Diagnostics $\mathrm{GmbH}$, Mannheim, Germany) was added to the cell lysis solution at $37^{\circ} \mathrm{C}$ for $30 \mathrm{~min}$ to digest plasmid DNA. Total HBV DNA replicative intermediates were purified from Huh7 cell lysates $96 \mathrm{~h}$ post-transfection and used as templates for qRT-PCR, which was conducted using SYBRGreen I nucleic acid stain (Roche Diagnostics $\mathrm{GmbH}$ ) on a LightCycler real-time thermal cycler (Applied Biosystems, Foster City, CA, USA) according to the manufacturers' instructions. Primers HBV-RC-F and HBV-RC-R (Table I) hybridized to the HBV surface gene were designed to quantify HBV-DNA relaxed circular (RC) genomes (100 bp fragments) by qRT-PCR relative to an external plasmid DNA standard.

Analysis of HBV DNA from intracellular core particles by Southern blot analysis. Replication competent HBV WT and MT plasmids were transfected into Huh7 cells. HBV DNA replicative intermediates from intracellular core particles were extracted and diluted in $40 \mu 1 \mathrm{Milli}-\mathrm{Q}$ water, $20 \mu \mathrm{l}$ of which were subjected to agarose gel electrophoresis, followed by denaturation and Southern blot analysis with ${ }^{32} \mathrm{P}$-labeled full-length HBV probes according to previously published protocols (24). Hybridization signals were visualized and analyzed by a phospho-imager (Cyclone ${ }^{\circledR}$ Plus Storage Phosphor System; Parkard Instrument Company, Inc., Meriden, CT, USA). Data of densitometry analyses were quantified using OptiQuant software (PerkinElmer, Waltham, MA, USA).

\section{Results}

HBV plasmid construction. A T1699C substitution in the $x$ gene, T2310C in ATG of the $\mathrm{P}$ open reading frame, C669A (rtL180M) and A741G (rtM204V) were ligated into pHBV1.3 to obtain pHBV1.3-XhoI, -XhoI-P-null, and -rtL180M/ M204V, respectively (Fig. 1A). Construction was carried out by fusion-PCR using primers carrying specific mutations and pHBV1.3 as the template. All of the molecular constructs were prepared using standard molecular biology techniques and their sequences were confirmed by restriction analysis and sequencing. The pHBV1.3 plasmid was digested with XhoI into 4782- and 1907-bp segments, while -XhoI and -XhoI-Pnull were cut into 4782-, 1568- and 339-bp segments (Fig. 2B).

Replication and protein expression of WT and MT plasmids. The plasmids, pHBV1.3, -XhoI, -XhoI-P-null, -rtM204V/I, and $-\mathrm{rtL} 180 \mathrm{M} / \mathrm{M} 204 \mathrm{~V}$, were transfected into the Huh7 cells. HBsAg and HBeAg in the culture medium were subjected to ELISA. Replicative intermediates of HBV from intracellular core particles were extracted from the Huh7 cells and detected by Southern hybridization in a standard manner. A segment (1176 bp) from 1023 to 2199 bp was PCR-amplified using the above extracted DNA as the template and P-XhoI-F1 and P-XhoI-R4 as primers with or without XhoI digestion. Judging by the result of SEAP activity in the culture medium, the 
Table I. Primer sequences for the construction of MT HBV plasmids and qRT-PCR.

\begin{tabular}{|c|c|c|}
\hline Name & Sequence $\left(5^{\prime} \rightarrow 3^{\prime}\right)$ & Amplicon size (bp) \\
\hline P-null-F1 & AATTGGAGCTCCACCGCGGACGAC & 1775 \\
\hline P-null-R4 & AGGTTGTGGAATTCCACTGCATGGCC & \\
\hline P-null-F2 & AGCCTATAGACCACCAAACGCCCCTA & 913 (down) \\
\hline P-null-R3 & TAGGGGCGTTTGGTGGTCTATAGGCT & 862 (up) \\
\hline P-RT-mt-F & TCTTCTCGAGGATTGGGGACC & 1257 \\
\hline P-RT-mt-R & GCAGCCATGGAAACGATGTAT & \\
\hline P-rtL180M-F & GTTTCTCATGGCTCAGTTTACTAG & 721 (down) \\
\hline P-rtL180M-R & CTAGTAAACTGAGCCATGAGAAAC & 557 (up) \\
\hline P-rtM204V/I-F & CTTTCAGTTAT $\boldsymbol{G}(\boldsymbol{C})$ TGGATGATGTGGT & 653 (down) \\
\hline P-rtM204V/I-R & ACCACATCATCCAC $(\boldsymbol{G})$ ATAACTGAAAG & 630 (up) \\
\hline $\mathrm{P}-X o h \mathrm{I}-\mathrm{F} 1$ & GCCCCTTTTACACAATGTGGTTATC & 1089 \\
\hline P-XohI-R4 & AGGCACCCCAGGCTTTACACTTTAT & \\
\hline $\mathrm{P}-X o h \mathrm{I}-\mathrm{F} 2$ & GTCTTTGAAGTATGCCTCGAGGTC & 503 (down) \\
\hline P-XohI-R3 & GACCTCGAGGCATACTTCAAAGAC & 673 (up) \\
\hline
\end{tabular}

MT, mutant; HBV, hepatitis B virus; qRT-PCR, quantitative real-time PCR; F, forward; R, reverse; underlining: restriction enzyme cutting site; double underlining: initiation codon which was mutated; bold text, italics: substitution mutation for mutant HBV plasmids construction; up/ down: upstream/downstream product size of fusion PCR.

A

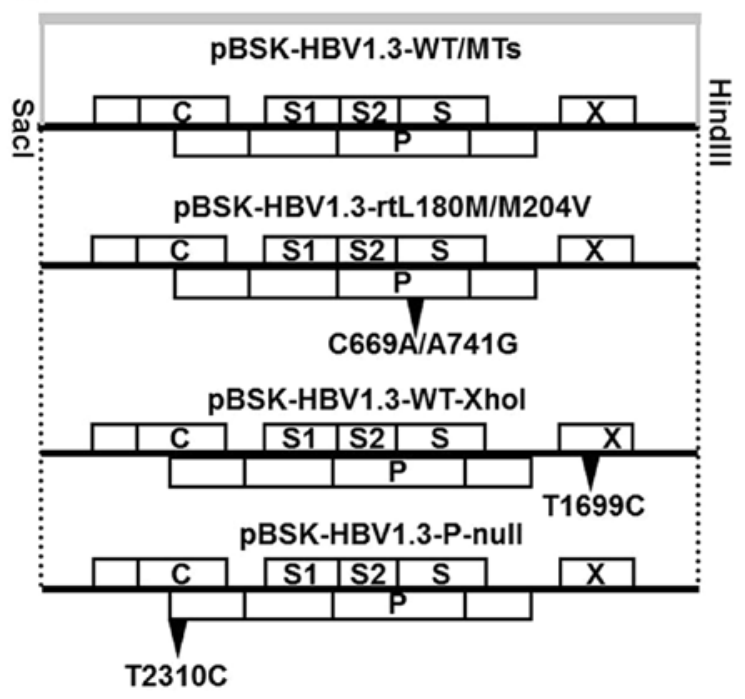

B

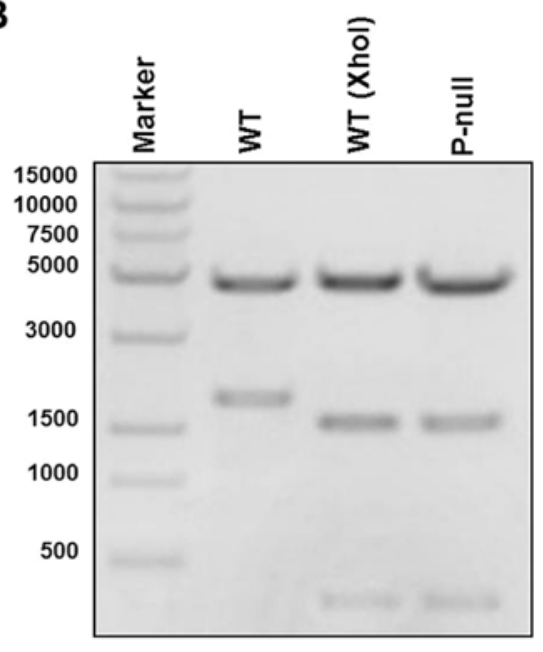

Figure 1. HBV replicon plasmids for cellular transfection of HBV genomes. (A) A schematic representation of substitutions used to generate MT HBV plasmids. Substitutions (C669A/A741G/T1699C/T2310C) were introduced into the HBV genome to generate pHBV1.3-rtL180M/M204V, -XhoI and -P-null. (B) Identification of additional XhoI sites in pHBV1.3-XhoI and -P-null. Approximately $1 \mu \mathrm{g}$ of plasmids of pHBV1.3,-XhoI and -P-null were digested by XhoI and then subjected to agarose gel electrophoresis.

transfection efficiencies of the HBV-bearing plasmids were comparative (data not shown). pHBV1.3-XhoI and -rtL180M/ M204V had similar HBV replication levels as the WT plasmid, while -rtM204V/I had moderately depressed HBV replication, and WT-XhoI-P-null terminated HBV replication (Fig. 2A, upper panel). All MT and WT plasmids expressed comparative HBs/eAg levels (Fig. 2E). The results from PCR and digestion agreed with those from Southern blot analysis, which showed that only the PCR products from WT-XhoI could be digested into 2 smaller segments of 673 and $503 \mathrm{bp}$.
Two different $H B V$ virions co-exist in individual Huh7 cells, but do not influence one another. In order to examine the interplay between different HBV virions co-existing in individual Huh7 cells, HBV replication-competent plasmids were co-transfected into Huh7 cells and their replication capacities were detected by PCR and XhoI digestion. The pHBV1.3rtA194T plasmid had a lower replication capacity and was not enhanced by pHBV1.3-XhoI and vice versa (Fig. 3C).

Furthermore, the non-replicative pHBV1.3-XhoI-P-null plasmid, in which the initiator polymerase codon was mutated, 


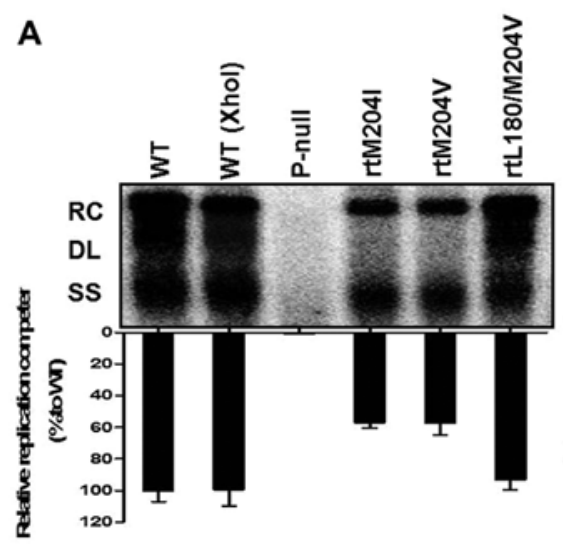

B
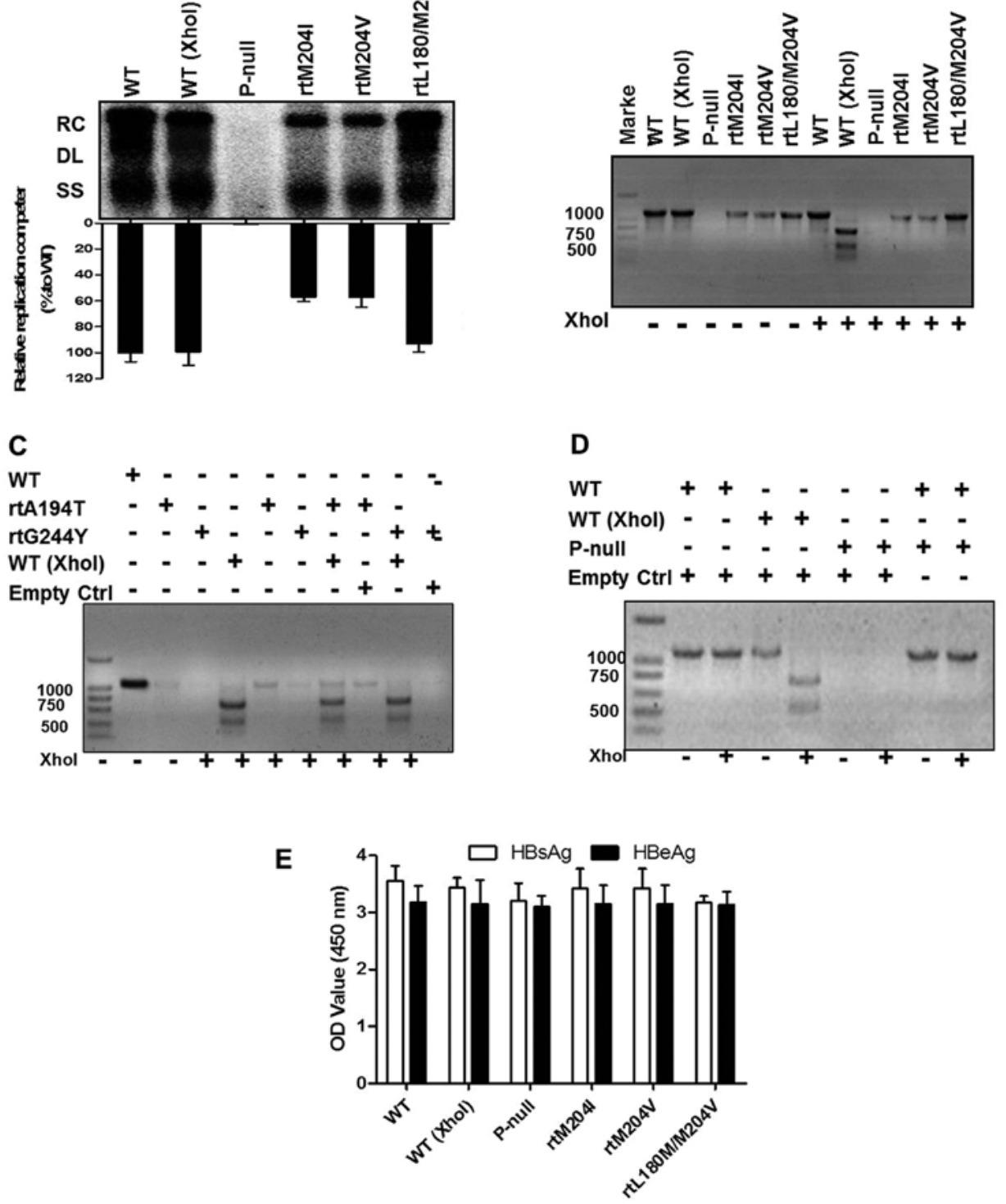

Figure 2. Replication capacity of WT and HBV mutants. Huh7 cells were transfected with WT or MT HBV plasmids. (A) Encapsulated viral DNA was extracted and detected by Southern blot analysis (upper panel). Relaxed circular (RC), double-stranded linear (DL), and single-stranded (SS) HBV DNAs are indicated. The relative level of WT HBV replication capacity is shown as a percentage of the control (black bars; lower panel). (B-D) Encapsulated viral DNA was extracted and detected by PCR using P-XhoI-F1 and P-XhoI-R4. PCR products with or without XhoI digestion were subjected to agarose gel electrophoresis. (E) HBsAg and $\mathrm{HBeAg}$ in the supernatant were detected using an ELISA diagnostic kit according to the manufacturer's instructions.

was co-transfected with pHBV1.3 into the Huh7 cells (Fig. 3D). No specific dissected bands were obtained from the pHBV1.3XhoI-P-null plasmid, indicating no replication rescue of pHBV1.3. These results were the precondition of our novel method constructed to analyze drug-resistant $\mathrm{HBV}$ phenotypes.

Southern blot analysis and real-time PCR to analyze drugresistant phenotypes of WT and $M T H B V$. The HBV plasmids, pHBV1.3, -XhoI and -rtL180M/M204V, were transfected into the Huh7 cells and treated with different concentrations of NAs. The intracellular encapsulated HBV genomes were extracted and subjected to Southern blot analysis (Fig. 3) and real-time PCR (Fig. 4) targeted to HBV core-associated DNA and RC DNA, respectively. The anti-HBV effect of NAs and susceptibility variation between the WT and MT plasmids were estimated. WT and WT (XhoI) were sensitive to LMV (Fig. 3A), LdT (Fig. 3B), ADV (Fig. 3C), ETV (Fig. 3D) and TDF (Fig. 3E). However, rtL180M/M204V led to reduced susceptibility to LMV and LdT and attenuated the amplitude of the former more so than that of the later, but maintained similar sensitivities to ADV, ETV and TDF in the WT plasmid. The same conclusion was also drawn by real-time PCR analysis (Fig. 4).

Novelmethodfortheanalysis of drug-resistant HBV phenotypes. In order to eliminate the diversity resulting from transfection efficiencies and to simulate the quasispecies in patients, we co-transfected cells with pHBV1.3-XhoI and -rtL180M/M204V followed by treatment with various NA concentrations. The intracellular intracellular encapsulated HBV genomes viral 


\section{A}
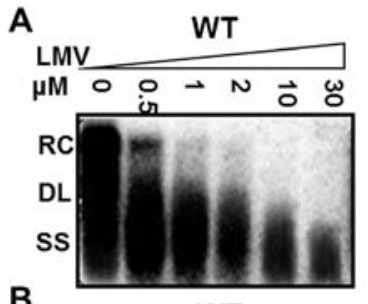

B
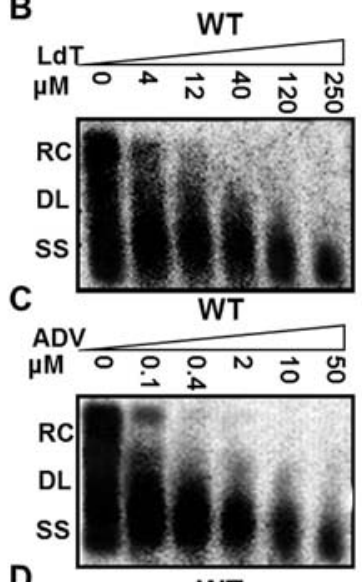

D
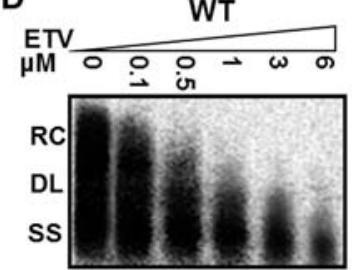

E
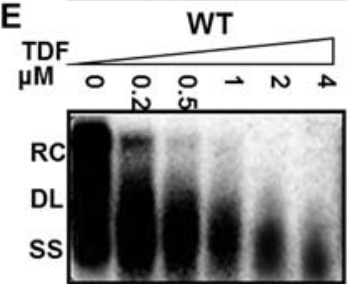
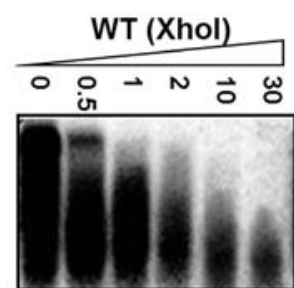

WT (Xhol)

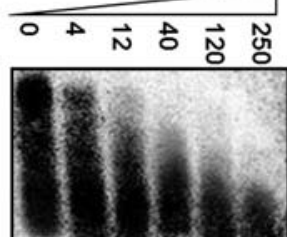

WT (Xhol)

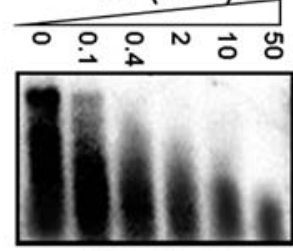

WT (Xhol)

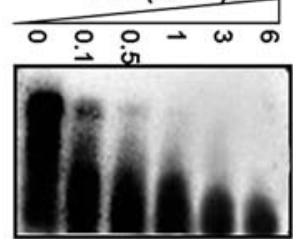

WT (Xhol)
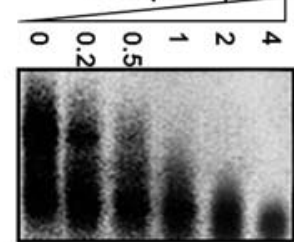

$\mathrm{rtL} 180 \mathrm{M} / \mathrm{M} 204 \mathrm{~V}$

0 ir $\rightarrow N \overrightarrow{0}$ o

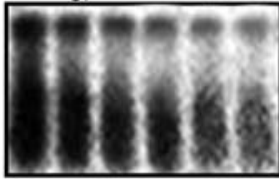

rtL180M/M204V
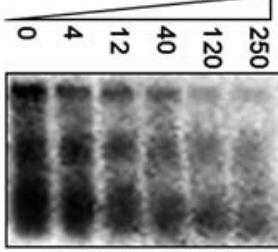

rtL180M/M204V
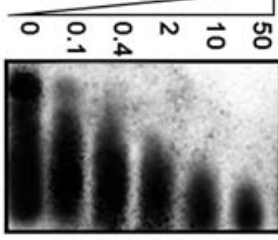

rtL180M/M204V
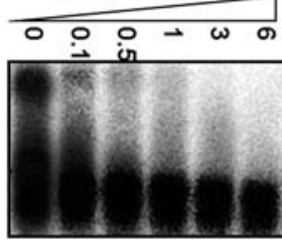

rtL180M/M204V
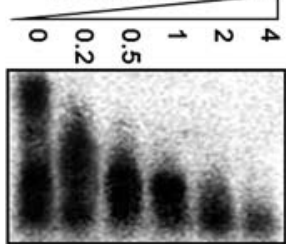

Figure 3. Southern blot analysis for the detection of the anti-HBV effects of 5 nucleos(t)ide analogues (NAs). Huh7 cells were transfected with pHBV1.3, -XhoI and rtL180M/M204V followed by treatment with (A) LMV, (B) LdT, (C) ADV, (D) ETV and (E) TDF at the indicated concentrations. Encapsulated HBV DNA was extracted and analyzed by Southern blot analysis.

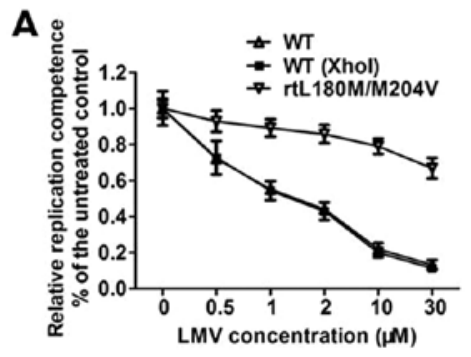

B :

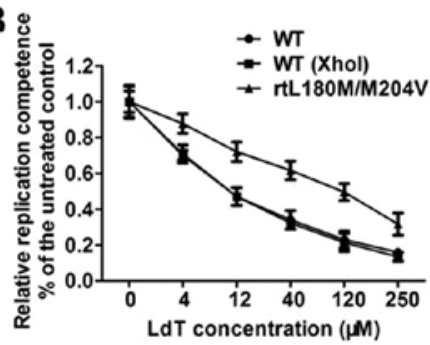

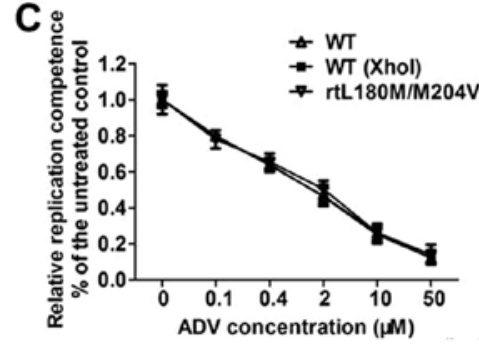

E

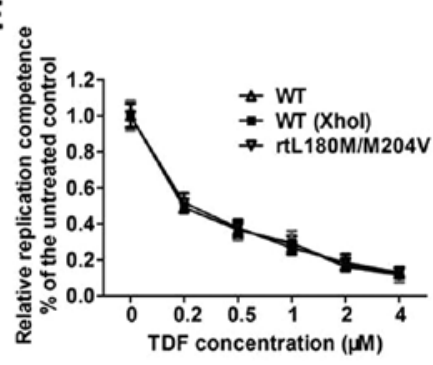

Figure 4. qRT-PCR was performed to detect the anti-HBV effects of 5 nucleos(t)ide analogues (NAs). Huh7 cells were transfected with pHBV1.3, -XhoI and $\mathrm{rtL180M/M204V.} \mathrm{Encapsulated} \mathrm{HBV} \mathrm{DNA} \mathrm{was} \mathrm{extracted} \mathrm{and} \mathrm{analyzed} \mathrm{by} \mathrm{qRT-PCR} \mathrm{to} \mathrm{evaluate} \mathrm{the} \mathrm{anti-HBV} \mathrm{effects} \mathrm{of} \mathrm{(A)} \mathrm{LMV,} \mathrm{(B)} \mathrm{LdT,} \mathrm{(C)} \mathrm{ADV,} \mathrm{(D)} \mathrm{ETV}$ and (E) TDF. The results shown were calculated as the means \pm SDs from 2 experiments $(n=3)$. 
A

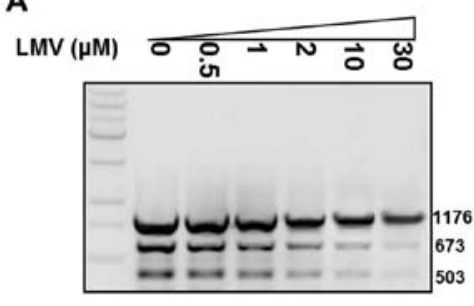

B

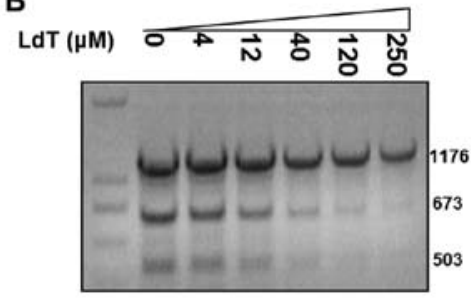

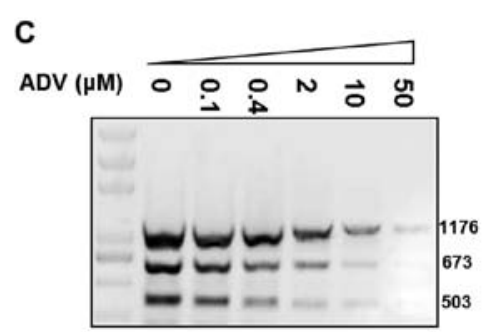

D

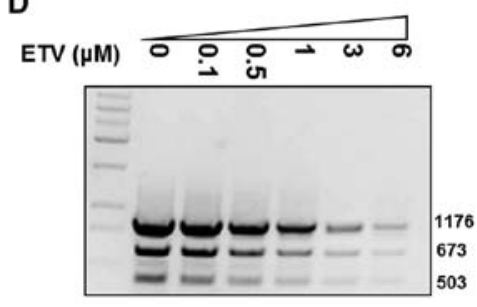

E

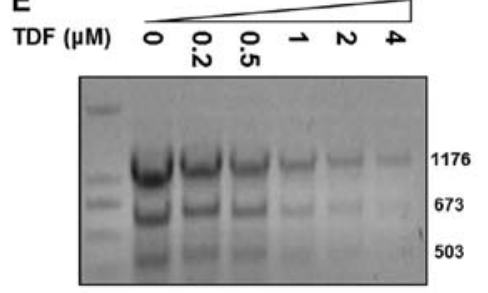

Figure 5. Resistance analysis was performed by PCR and XhoI digestion. The pHBV1.3-XhoI plasmid was co-transfected with -rtL180M/M204V into Huh7 cells and 5 nucleos(t)ide analogues (NAs) were added. The mixture of encapsulated HBV DNA was extracted and used as the template to amplify the fragment from 1023 to $2199 \mathrm{bp}$. The PCR products were digested by XhoI and subjected to agarose gel electrophoresis. The densitometry of 1076-bp bands represents the replication level of pHBV1.3-rtL180M/M204V at the indicated drug concentrations. The 503- and 673-bp bands comprised the WT plasmid. The changes in relevant band intensities were used to evaluate the susceptibility of pHBV1.3-XhoI and -rtL180M/M204V to (A) LMV, (B) LdT, (C) ADV, (D) ETV and (E) TDF.

genomes composed of pHBV1.3-XhoI and -rtL180M/M204V were extracted and distinguished by PCR and XhoI digestion. Only the products from the former could be cut into 2 sections by XhoI. Hence, the susceptibility difference to a given drug could be demonstrated by the change in specific band segments through agarose electrophoresis. ADV, ETV and TDF significantly inhibited all 3 segments (Fig. 5A and C), while LMV and LdT only inhibited the 503- and 673-bp segments and the 1176-bp segment composing rtL180M/M204V was suppressed more slowly than the 503- and 673-bp segments composing -XhoI. These results demonstrated that $\mathrm{rtL} 180 \mathrm{M} / \mathrm{M} 204 \mathrm{~V}$ was resistant to $\mathrm{LMV}$ and $\mathrm{LdTl}$, and are in accordance with the results shown in Fig. 4 and those in previous reports. This novel method was successfully established for the analysis of drugresistant HBV phenotypes.

\section{Discussion}

As reported previously, due to the absence of HBV-RT proofreading capacity and its high replication rate, $\mathrm{HBV}$ exists as a quasispecies composed of a number of mutants that possess different fitness levels in certain environments. Due to the advantage of competitive replication, mutants with higher fitness levels may predominate in the total population and may differ in changing environments, such as the immune state of patients and treatment with interferon or NAs. As shown in previous studies, non-replicate HBV-RT mutations can be rescued by eukaryotic expression plasmids of HBV polymerase $(27,28)$. In HBV quasispecies, it is unclear whether mutants with low replication capacity may be restored by a polymerase produced by mutants with normal replication capacities. In this study, we did not find this restoring phenomenon when viruses with various replication capacities co-existed.

HBV-RT mutants with resistance to NAs are selected during long-term antiviral therapy; hence, these mutations will lead to virological breakthrough and therapeutic failure. Much research has been focused on drug-resistant mutations of HBV. The discovery of mutations for the analysis of novel resistance phenotypes is crucial and necessary for guiding the future development of antiviral medications.

The traditional detection method involves isolating HBV sequences from CHB patients and analyzing them for virological breakthrough to find drug-resistant mutations, which are then introduced into HBV replicon plasmids and transfected into Huh7/HepG2 cells in parallel with WT HBV and adding different NA concentrations to the culture medium. Susceptibilities to given drugs are then evaluated by Southern blot analysis or real-time PCR. Mutations that reduce susceptibility to a given drug are designated as resistance mutations. The novel method established in this study allowed for the more manageable and more rapid analysis of resistance phenotypes using regular PCR and restriction enzyme digestion. Certainly, PCR-digestion-based assay to distinguish HBV replication differences and susceptibility changes is not as convincing as ${ }^{32} \mathrm{P}$-labeled Southern blot analysis. Only 20 cycles of PCR were run to minimize the deviation resulting from PCR amplification. As shown in Fig. 2A-D, PCR-digestion-based assay was successful in distinguishing the replication capacity and susceptibility differences of mutants and WT HBV to a given drug, which is in line with the Southern blot analysis results. Furthermore, ${ }^{32} \mathrm{P}$-labeled Southern blot analysis, which is harmful to the environment and laboratory technicians, was avoided. Importantly, our co-transfection system allowed for a similar transfection efficiency of different plasmids and the co-existence of different virions in individual hepatocellular carcinoma cells to closely mimic actual quasispecies. Therefore, the replication capacity of WT (XhoI) and MT HBV can be successfully distinguished using greyscale analysis. 
In the future, we aim to refine this co-transfection system to achieve better standardization in vitro and aim to apply this method in vivo using a mouse model by hydrodynamic injection of an engineered, replication-competent HBV plasmid DNA into the tail veins of C57BL/6 mice.

\section{Acknowledgements}

We are grateful to Xinwen Chen and Mengji Lu for excellent technical support and guidance. This study was supported by the Qianjiang Talents Project of the Technology Office of Zhejiang Province, China (no. 2012R10084).

\section{Referemces}

1. Lavanchy D: Hepatitis B virus epidemiology, disease burden, treatment, and current and emerging prevention and control measures. J Viral Hepat 11: 97-107, 2004.

2. Lee WM: Hepatitis B virus infection. N Engl J Med 337: 1733-1745, 1997.

3. Huang LR, Wu HL, Chen PJ and Chen DS: An immunocompetent mouse model for the tolerance of human chronic hepatitis B virus infection. Proc Natl Acad Sci USA 103: 17862-17867, 2006.

4. Amini-Bavil-Olyaee S, Herbers U, Sheldon J, Luedde T, Trautwein C and Tacke F: The rtA194T polymerase mutation impacts viral replication and susceptibility to tenofovir in hepatitis B e antigen-positive and hepatitis B e antigen-negative hepatitis B virus strains. Hepatology 49: 1158-1165, 2009.

5. Zoulim F and Locarnini S: Hepatitis B virus resistance to nucleos(t)ide analogues. Gastroenterology 137: 1593-1608, 2009.

6. von Kleist M, Metzner P, Marquet R and Schutte C: HIV-1 polymerase inhibition by nucleoside analogs: cellular- and kinetic parameters of efficacy, susceptibility and resistance selection. PLoS Comput Biol 8: e1002359, 2012.

7. Galmarini CM, Mackey JR and Dumontet C: Nucleoside analogues: mechanisms of drug resistance and reversal strategies. Leukemia 15: 875-890, 2001.

8. Sabini E, Hazra S, Konrad M and Lavie A: Nonenantioselectivity property of human deoxycytidine kinase explained by structures of the enzyme in complex with L- and D-nucleosides. J Med Chem 50: 3004-3014, 2007.

9. Liu F, Chen L, Yu DM, et al: Evolutionary patterns of hepatitis B virus quasispecies under different selective pressures: correlation with antiviral efficacy. Gut 60: 1269-1277, 2011.

10. Nishijima N, Marusawa H, Ueda Y, et al: Dynamics of hepatitis B virus quasispecies in association with nucleos(t)ide analogue treatment determined by ultra-deep sequencing. PloS One 7: e35052, 2012.

11. Brunelle MN, Jacquard AC, Pichoud C, et al: Susceptibility to antivirals of a human HBV strain with mutations conferring resistance to both lamivudine and adefovir. Hepatology 41: 1391-1398, 2005
12. Levine S, Hernandez D, Yamanaka G, et al: Efficacies of entecavir against lamivudine-resistant hepatitis B virus replication and recombinant polymerases in vitro. Antimicrob Agents Chemother 46: 2525-2532, 2002.

13. Pallier C, Castera L, Soulier A, et al: Dynamics of hepatitis B virus resistance to lamivudine. J Virol 80: 643-653, 2006.

14. Tenney DJ, Levine SM, Rose RE, et al: Clinical emergence of entecavir-resistant hepatitis B virus requires additional substitutions in virus already resistant to Lamivudine. Antimicrob Agents Chemother 48: 3498-3507, 2004.

15. Tenney DJ, Rose RE, Baldick CJ, et al: Long-term monitoring shows hepatitis B virus resistance to entecavir in nucleosidenaive patients is rare through 5 years of therapy. Hepatology 49: 1503-1514, 2009.

16. Walsh AW, Langley DR, Colonno RJ and Tenney DJ: Mechanistic characterization and molecular modeling of hepatitis B virus polymerase resistance to entecavir. PloS One 5: e9195, 2010.

17. Liu Y, Wang CM, Cheng J, et al: Hepatitis B virus in tenofovirnaive Chinese patients with chronic hepatitis B contains no mutation of rtA194T conferring a reduced tenofovir susceptibility. Chin Med J 122: 1585-1586, 2009.

18. Yuen MF and Lai CL: Treatment of chronic hepatitis B. Lancet Infect Dis 1: 232-241, 2001

19. Lee YS, Suh DJ, Lim YS, et al: Increased risk of adefovir resistance in patients with lamivudine-resistant chronic hepatitis B after 48 weeks of adefovir dipivoxil monotherapy. Hepatology 43: 1385-1391, 2006.

20. Jeon JW, Shin HP, Lee JI, et al: Efficacy of entecavir and adefovir combination therapy for patients with lamivudine- and entecavirresistant chronic hepatitis B. Dig Dis Sci 57: 1358-1365, 2012.

21. Svicher V, Cento V, Salpini R, et al: Role of hepatitis B virus genetic barrier in drug-resistance and immune-escape development. Dig Liver Dis 43: 975-983, 2011.

22. Lei YC, Hao YH, Zhang ZM, et al: Inhibition of hepatitis B virus replication by APOBEC $3 \mathrm{G}$ in vitro and in vivo. World $\mathrm{J}$ Gastroenterol 12: 4492-4497, 2006.

23. Wu C, Deng W, Deng L, et al: Amino acid substitutions at positions 122 and 145 of hepatitis B virus surface antigen (HBsAg) determine the antigenicity and immunogenicity of HBsAg and influence in vivo HBsAg clearance. J Virol 86: 4658-4669, 2012.

24. Qin B, Budeus B, Cao L, et al: The amino acid substitutions rtP177G and rtF249A in the reverse transcriptase domain of hepatitis B virus polymerase reduce the susceptibility to tenofovir. Antiviral Research 97: 93-100, 2013.

25. Qiu J, Qin B, Rayner S, et al: Novel evidence suggests Hepatitis B virus surface proteins participate in regulation of HBV genome replication. Virol Sin 26: 131-138, 2011.

26. Kain SR: Use of secreted alkaline phosphatase as a reporter of gene expression in mammalian cells. Methods Mol Biol 63: 49-60, 1997.

27. Blum HE, Galun E, Liang TJ, et al: Naturally occurring missense mutation in the polymerase gene terminating hepatitis B virus replication. J Virol 65: 1836-1842, 1991.

28. Heipertz RA Jr, Starkey JL, Miller TG, et al: trans-Complementation of HBV rtM204I mutant replication by HBV wild-type polymerase. Virology 388: 57-67, 2009. 